\title{
MINIMAL RESIDUAL DISEASE IN ACUTE LYMPHOBLASTIC LEUKEMIA IN THE CONTEXT OF HEMATOPOETIC STEM CELL TRANSPLANTATION
}

\author{
Maura Rosane Valério Ikoma-Colturato, Md Phd \\ Affiliation: Amaral Carvalho Hospital, Sabin Medicina Diagnóstica
}

Correspondence to: Maura Ikoma - msikoma@uol.com.br

\begin{abstract}
Minimal or measurable residual disease (MRD) is considered the most important independent prognostic factor in acute lymphoblastic leukemia (ALL). MRD status after clinical remission has been used to establish the risk of relapse and therapeutic stratification, identifying patients who can benefit from therapeutic intensification, including allogeneic stem cell transplantation (alloSCT). The pre alloSCT MRD also identifies patients eligible for transplant and those with low or high risk of relapse after transplantation, according to the level of MRD detected. However, MRD status post-alloSCT has been shown to be a more powerful predictor of relapse than pre-transplant MRD. In addition, it is important to take into account that there are some factors to be considered to better interpret MRD information, these include: the method used for MRD assessment and its sensitivity and specificity, which may vary according to each specific time point of evaluation; the treatment regimen used; and the identification of genetic lesions that combined with MRD information can further improve the management of patients with ALL.
\end{abstract}

Key Words: Minimal residual Disease (MRD), Acute Lymphoblastic Leukemia (ALL), allogeneic Stem Cell Transplantation (allo-SCT)

\section{INTRODUCTION}

Minimal or measurable residual disease is, by definition, a sub-microscopic disease that can be detected by sensitive methods that more accurately monitor disease kinetics during and after the treatment of hematological malignancies. MRD quantification can assess the response to treatment of individual patients by the magnitude of the disease burden reduction and establish the risk of disease relapse. In acute lymphoblastic leukemia, MDR is considered the most important predictable relapse factor and it has been widely used by most cooperative ALL treatment groups to guide treatment decisions.1-11 The MRD level can identify patients who need treatment intensification, including with allogeneic SCT (alloSCT). Here, the impact of the status of peri and post-transplant MRD in patients with ALL will be discussed, as well as some aspects that must to be taken into account for a better interpretation of the results of MRD by clinical hematologists.

\section{PROGNOSTIC SIGNIFICANCE OF MRD}

MRD assessment at the end of induction therapy is useful to identify patients with a low risk of relapse. Any persisting MRD level at the end of consolidation therapy is associated with a high risk of relapse and indicates the need for therapy intensification. $[9,10,12,13]$

The MRD persistence at $\geq 10-3$ before $S C T$ in children with high-risk relapsed ALL reflects a disease which is highly resistant to conventional intensive chemotherapy, and which requires prospective investigation of 
new treatment strategies with innovative targeted or immunotherapeutic approaches.[14]

Many studies have demonstrated the prognostic impact of MRD pre- and post-alloSCT. A study by the BFM group included children and adolescents with relapsed $A L L$, eligible to receive alloSCT in $\geq$ second remission (CR2). The methods used for MRD detection included both multiparametric flow cytometry (MFC) and quantitative real time PCR (RTqPCR). The MRD cutoff value of less than 10-4 leukemic cells turned out to be a feasible discriminator between patients at high ( $\geq 10-4$ leukemic cells) or low risk $(<10-4$ leukemic cells) for subsequent relapse. In other words, patients who underwent transplantation with an MRD < 10-4 leukemic cells, had a higher event free survival (EFS) and lower cumulative incidence of relapse (CIR) than those who underwent alloSCT with an MRD load of $\geq 10-4$ leukemic cells. [15]

However, a meta-analysis study showed that although positive MRD (MRD+) before alloSCT was a significant negative predictive factor of relapse-free survival (RFS), EFS and overall survival (OS), a MRD+ result prior to transplantation was not associated with a higher rate of non-relapse mortality. [16]

Based on MRD status, patients stratified as high-risk of relapse have been shown to benefit from alloSCT, but the maintenance of MRD positivity after transplantation correlates with a poor outcome. $[13,17-$ 20] Retrospective studies with pediatric and adult ALL patients have shown that patients with undetectable MRD by MFC or by RTqPCR before myeloablative alloSCT had a better outcome than patients with any level of MRD+. Patients with MRD+ after transplantation had significantly worse outcomes than patients with undetectable MRD after transplantation. [10, 3] and 10-4 were the minimum discriminatory MRD detection limits used in these studies. [13,21] Similar results were observed using a more sensitive method for MRD detection such as next generation sequencing (NGS), in which any post-SCT NGS-MRD positivity resulted in an increased risk of relapse whereas the absence of detectable NGS-MRD pre-SCT defined good-risk patients. 22 In addition, patients who converted from MRD+ to MRD-negative after transplant had been in remission for at least two years after alloSCT.13 The kinetics of MRD by MFC in pediatric patients with ALL in the peri-haploidentical SCT was also important in predicting the risk of relapse. [23]

A multicenter study in pediatric ALL patients compared the prognostic value of pre-alloSCT and post-alIOSCT MRD kinetics. Definitions of MRD status were: undetectable MRD was considered as MRD negative; detectable MRD $<10-4$ (RTqPCR) or $<0.01 \%$ (MFC) was MRD low positive; MRD $\geq 10-4$ to $<10-3$ (RTqPCR) or $\geq 0.01$ to $<0.1 \%$ (MFC) was MRD high positive; and MRD $\geq 10-3$ (RTqPCR)) or $\geq 0.1 \%$ (MFC) was MRD very high positive. They demonstrated that patients with detectable MRD pre-SCT and MRD post-SCT had a significantly lower EFS and higher CIR, especially those with higher MRD levels. But there was no effect on outcomes when MRD pre-SCT was detected at the lowest levels $(<10-4)$ in patients who achieved post-SCT undetectable MRD. However, after transplantation even low levels of MRD were always highly predictive of relapse $(p=0,001)$. Furthermore, any detectable MRD level on days +180 and +365 was highly predictive of relapse and poor survival. Conversely, patients who were MRD negative on day +365 had long-term survival. In conclusion, the risk of relapse was more strongly influenced by MRD post-SCT than by MRD pre-SCT. [19]

On the other hand, MRD monitoring is much less frequently used after alloSCT because chimerism monitoring provides an alternative for early relapse detection. However, there is evidence that Ig/TCRbased MRD has higher sensitivity and specificity compared with chimerism analysis. [24]

Time points for MRD assessment in ALL patients eligible for allo-SCT

MRD levels at different time points have different prognostic values for relapse. The most suitable time points for MRD assessment are not consensual, however, the following studies can guide clinical strategies in patients with ALL.

\section{Pre allo-SCT MRD assessment}

One study has demonstrated the kinetics of MRD reduction in high-risk relapsed ALL patients before alloSCT are heterogeneous. The study noted that patients achieved a rapid or slow reduction in MRD during the treatment period from induction therapy to directly before transplantation. Some patients who initially had a deeper therapeutic response experienced an increased in the MRD level during this period. Therefore, the study concluded that MRD assessments should be done early before consolidation therapy and before each chemotherapy cycle, including immediately before alloSCT. [14] In another prospective study in children with relapsed ALL treated according to the BFM study protocols, MRD was measured by RTPPCR, at a median of 13 days before alloSCT to assess the prognostic significance of MRD before transplantation. [15] 
MRD was assessed by RTqPCR (BCR-ABL1 transcript with 10-5 sensitivity) within 30 days before allo-SCT, in patients who received chemotherapy combined with tyrosine-kinase inhibitors (TKIs) before transplantation. Undetectable MRD was one of the factors most influential in RFS for adult patients with Philadelphia+ $(\mathrm{Ph} 1+)$ ALL transplanted in first clinical remission $(C R 1)(p=0.0004)$. [25]

These studies indicate that MRD assessment should be done very close to alloSCT.

\section{Post allo-SCT MRD assessment}

The accuracy of MRD measurements (by MFC and RT-pPCR) in predicting relapse was investigated at days $+30,+60,+90$, and +180 post-SCT. From day +60 onwards, the discriminatory power of MRD detection was greater to predict the probability of relapse.18 On the other hand, especially at earlier times after transplantation (day +30$)$, the detection of NGS-MRD after SCT, was better in the prediction of relapse than MFC-MRD ( $p<0.0001)$. [22]

The evaluation of factors which may impact the outcome in pediatric patients with ALL undergoing alIOSCT, such as MRD+ pre SCT, the status of remission (CR2, CR3), non-TBI conditioning regimen, absence of aGVHD by day+190 post-transplant, can define groups with a high risk of relapse who can benefit from the more frequent MRD assessment and early therapeutic intervention. [19]

\section{EVALUATING AN ALL MRD RESULT}

Some important information must be considered and added to MRD results to refine outcome prediction in ALL patients, such as the leukemia biology, the sensitivity of the method used for MRD detection, the time points of assessment and the treatment regimen used.

\section{Methods of MRD detection}

Knowledge of the characteristics and limitations of each method is essential for the correct interpretation of MRD results. The sensitivity and specificity of methods for measuring MRD are different and vary during ALL treatment. This means that MRD detection is influenced by the method used at each given evaluation time point.

Molecular methods include the use of RTqPCR to detect leukemia-specific or patient-specific molecular markers, such as fusion gene transcripts and immunoglobulin / T cell receptor (Ig / TCR) gene rearrangements. Multiparametric flow cytometry is based on the detection of "different from normal" immunophenotypes. These methods reach limits of 10-4 to 10-5 for MRD detection, which means 1 leukemic cell in 10.000 to 100.000 normal cells. Recently, new high-throughput technologies to quantify MRD have been introduced: NGS for Ig/TCR rearrangements.22,26 and next generation flow (NGFlow) based on immunophenotyping.27 These reach limits of detection of 10-6 to 10-7 (1: 1.000 .000 to 1: 10.000 .000 normal cells) and 10-5 to $10-6$ respectively.

It must be emphasized that, although these methods reach high sensitivity, a negative result of MRD does not necessarily mean eradication of the disease, rather, that the disease burden may be below the detection limit of the method used. [28]

\section{Molecular methods of MRD detection}

Both $1 \mathrm{~g} / \mathrm{TCR}$ RTqPCR and $\mathrm{Ig} / \mathrm{TCR}$ NGS require a diagnostic sample as a reference to identify the leukemia-specific index rearrangements that are monitored throughout therapy. False negative MRD results may occur using RTpPCR/ IgTCR rearrangements due to clonal evolution in immature leukemic blasts, which can lead to the loss of the leukemia-specific lg / TCR sequence. On the other hand, false positive MRD results can be a consequence of massive bone marrow regeneration after treatment which can cause nonspecific annealing of the primer. [28]

Although well standardized, assessment of MRD by RTq PCR/ IgTCR rearrangements is time consuming and labor intensive, requiring technical expertise. [29]

The NGS of the $\mathrm{Ig} / \mathrm{TCR}$ gene rearrangements can overcome some of the limitations of RTqPCR and can increase sensitivity, provided that sufficient numbers of cells are analyzed.

NGS does not require the construction of patient-specific oligonucleotides, because the same multiplex PCR assay can be used to identify and follow-up the index sequence. $[28,30]$ NGS also offers the advantage of being able to track minor subclones, responsible for driving relapse, which may not be identified by other methods. A disadvantage of NGS-based MRD detection is the need for large amounts of cells / DNA that can limit its usefulness. This often represents a serious limitation in the aplastic samples during treatment. $[28,30]$

NGS is still not well standardized and clinically validated, although there is evidence that NGS is more sensitive to identifying clinically significant MRD than other methods. $[20,22,26,29]$ For example, NGS- 
MRD post-alloSCT has been shown to be more predictive of relapse and survival than MFC-MRD, suggesting a role for this technique in defining patients who would be eligible for post-transplant interventions. [22]

Both molecular methods are expensive and are not widely available in Brazil.

RTqPCR is also used to detect MRD in patients Ph1+ $A L L$, detecting the BCR-ABL1 fusion gene with a detection threshold in the range10-4 to 10-5. One disadvantage of this method is that these PCR assays in which the p190 transcript is present are not fully standardized like p210 for Chronic Myeloid Leukemia, which makes it difficult to interpret the results.30 RTq PCR Ig / TCR rearrangements may be more specific than BCR-ABL1 for MDR monitoring in patients with Ph1+ ALL.30,31 MRD measured by MFC and/or RTpPCR produced largely equivalent results in a threshold of $0.01 \%$, which is the limit used to define MRD positivity in Ph1+ALL patients. Both methods have been proven to provide a more accurate quantification of residual leukemic cells than BCR-ABL1 transcripts. [31]

However, there are conflicting data: studies comparing BCR-ABL1 MRD and Ig/TCR MRD demonstrated significant differences in detection, in favor of BCR$A B L 1$ fusion transcript.28 It seems that Ig/TCR and $B C R-A B L 1$ MRD may provide distinct insights into MRD kinetics of different leukemic subpopulations in response to therapy. [28,30-32]

Despite the potential disadvantages, PCR for BCR$A B L 1$ is the recommended method for MRD assessment of Ph1+ ALL in the North American consensus, because it is superior to conventional MFC in predicting outcomes in this ALL subtype. [30]

\section{MRD by multiparametric flow cytometry}

MFC is based on the identification of leukemia-associated immunophenotypes (LAIPs) and the differences in blast cell immunophenotypes in relation to the maturation patterns of their normal counterparts. MFC is faster compared to molecular methods, which makes it useful for immediate therapeutic decisions. Indeed, MFC is less labor intensive and more widely available than PCR methods. MFC has high applicability (LAIPs can be identified in more than $90 \%$ of patients with ALL) and do not require information about the diagnostic immunophenotype. $[28,30]$

Sensitivity of conventional MFC-MRD detection is about 1 log lower than that for the molecular methods
(10-4), [12,33] although the concordance between the paired RTpPCR and MFC-MRD results has been demonstrated in children and in adults. $[19,31,34]$

MFC performance can be influenced by the similarities between leukemic lymphoblasts and regenerative lymphoid precursors. [35] In addition, phenotypic shifts that occur in residual leukemic cells, as well as in normal regenerative cells during therapy, can lead to false-negative or false-positive results. [35]

The conventional MFC-MRD has limited interlaboratory standardization, which makes the interpretation of results susceptible to the experience of each flow cytometry analyst. As a result, this heterogeneity of approaches to MRD detection generates the differences in sensitivity and specificity of tests among laboratories. The development of NGFlow, a fully standardized MFC technology, can decrease the subjectivity of the interpretation of MRD assays, reaching high sensitivity of the test (10-6) which is directly related to the number of cells ( $>5$ million) analyzed. $[27,29]$ Like other methods, NGFlow MRD requires training and knowledge. [29] Therefore, MFC-MRD requires significant technical expertise [28-30]

Anti-CD19 therapies influence the detectability of residual leukemic cells, due to the partial or total loss of the main markers for the detection of MRD of $B$ cell precursor (BCP)- ALL. [36] Different approaches are necessary for the detection of MRD in the context of anti-CD19 immunotherapies. [36] However, there is no consensus on the best MFC strategies for this purpose.

In summary, conventional MFC and RTpPCR can achieve sensitivity levels similar to those of NGS, up to a detection limit of 10-4 for MRD assessment, but NGS can achieve a higher degree of sensitivity and specificity than both. $[20,28,30]$ However, NGFlow has been shown to achieve similar sensitivity to RTpPCR in the MRD of BCP-ALL.27 To date, there have been no comparative studies on the sensitivity between NGS and NGFlow.

\section{Genetic factors and MRD response}

Genetic abnormalities associated with some subtypes of ALL are significantly associated with MRD status during treatment. [37] Adult patients with Philadelphia-like ALL, KMT2A -MLL gene rearrangement, and early T-cell precursor ALL (ETP-ALL) appear to have relatively poor outcomes regardless of MRD status (at a sensitivity level of 10-4). These disease subtypes are also more likely to have persistent MRD, despite intensive therapy.30 Patients with high risk cytogenetics are generally associated with poor 
outcomes, even achieving a good response with undetectable MRD at any moment during treatment. In a cohort of 3113 patients treated on UKALL2003, the distributions of MRD results at the end of induction therapy were different in groups of patients with different genetic subtypes $(p<0.001)$. [38] Patients with good-risk cytogenetics (ETV6-RUNX1, high hyperdiploidy) demonstrated faster clearance of leukemic cells (MRD by PCR Ig/TCR rearrangement with a limit of detection of $1 \times 10-5)$, while patients with high-risk cytogenetics (iAMP21, KMT2A rearrangement, haploid/ hypodiploid) and T-cell acute lymphoblastic leukemia responded more slowly. [38] Intermediate-risk patients who had genetic heterogeneity and variable MRD kinetic: TCF3-PBX1 or $\mathrm{t}(1 ; 19)$ exhibited a fast disease clearance, but these patients needed more intensive therapy to avoid relapses. [38,39] Other BCP- ALL with normal or abnormal cytogenetics, and also alterations of copy number, such as ABLclass fusions, JAK-STAT abnormalities, IKZF1 deletion, IKZF plus usually have slower disease clearance with prolonged persistence of MRD. [38] Although the risk of relapse is directly proportional to the level of MRD in each genetic risk group, the absolute risk of relapse associated with a specific level of MRD varies according to the genetic subtype. The integration of genetic information and MRD results can improve risk algorithms for treatment decisions. [38-41]

Hypodiploidy: in a retrospective cohort, the Children's Oncology Group observed that alloSCT has no impact on the outcome of children and young adults with hypodiploid BCP-ALL in CR1. Patients with MRD $<0.01 \%$ by MFC at end of induction therapy had 5 -year EFS of $66.3 \% \pm 7.9 \%$ with alloSCT $(n=39)$ and $60.3 \pm 9.2 \%$ without $(n=35 ; p=0.77)$. Five-year OS was $79.5 \% \pm 6.7 \%$ with SCT and $66.7 \% \pm 8.8 \%$ without $(p=0.39)$. Furthermore, CIR did not differ significantly between chemotherapy and SCT groups $(p=0.22)$ [42].

KMT2A (MLL) rearrangements: these occur more frequently in BCP- ALL, but also in a small fraction of T-ALL (5-10\% of T-ALL patients), mainly in pediatric patients ( $80 \%$ infants), in different proportions and types of molecular lesions. [39,43] The presence of these molecular signatures associated with MRD status determines a high proportion of refractory diseases, despite intensive therapies. $[30,38,39]$

$B C R-A B L-l i k e$ or Philadelphia like ALL: is a subgroup of BCP -ALL which has a gene expression profile similar to that of BCR-ABL1-positive ALL, with a high frequency of IKZF1 alterations, but lacking the BCR-ABL1 fusion protein. This subtype comprises $10 \%$ of the cases of BCP-ALL in children and $25 \%$ of the cases of ALL in adolescents and young adults. [39] The spectrum of genetic alterations is diverse, including rearrangements involving tyrosine kinase genes such as ABL and PDGFR, which respond to TKI. $[44,45]$ Other rearrangements target JAK and EPOR, which are sensitive to JAK inhibitors in preclinical studies. [46] In addition, rearrangements involving the cytokine receptor gene CRLF2, which were identified in $50 \%$ of patients with BCR-ABL1 like ALL, are often associated with JAK mutations and also potentially sensitive to JAK inhibition. $[47,48]$ In most studies, CRLF2 rearrangements are associated with a poor prognosis, particularly in cases with concomitant IKZF1 alterations. [48] However, risk-oriented therapy, including intensive chemotherapy with or without alloSCT based on the level of MRD during induction therapy, can eliminate the poor prognosis of this group of patients. [33]

IKZF1 deletions: these also occur in a subset of patients with poor-response, high-risk ALL without any known chromosomal rearrangement IKZF1 plus is characterized by IKZF1 deletions co-occurring with other copy number alterations. [39] IKZF1 plus had no prognostic impact in patients with undetectable MRD after induction therapy, but in patients with persistent positive MRD, they faced a 10-fold higher relapse rate in stratified analyses by MRD levels, describing a very poor and MRD-dependent prognostic profile in BCP-ALL [49]

CRLF2 rearrangements: these are also observed in $50 \%$ of ALL patients with Down syndrome, responsible for the inferior outcome due to the increased risk of relapse. In addition, these patients also have high rate of treatment-related mortality. [39,48]

ALL with intrachromosomal amplification of chromosome 21 (iAMP 21): this is considered an ALL subtype of high-risk cytogenetics and requires an intensive treatment modality. $[38,50,51]$ Intensification of chemotherapy has ended the poor prognosis once associated with this ALL subtype. [50] The BFM group considered that MRD alone identifies high-risk patients with iAMP21. [52]

Philadelphia chromosome (BCR-ABL1)- Ph1+ ALL: this occurs in about $3 \%$ of children with ALL and has been considered associated with poor outcome, despite intensive chemotherapy regimens and alloSCT. The introduction of TKI has markedly improved outcomes, avoiding alloSCT in MRD negative patients, but relapse remains the main cause of treatment failure. [31,53] MRD kinetics in children with $\mathrm{Ph} 1+\mathrm{ALL}$ who reached MRD $\leq 10-4$ leukemic cells at the end of induction therapy, evaluated by 
RTqPCR (Ig/TCR and BCR-ABL1 fusion transcript with sensitivity of 10-4) [53] or by MFC and Ig/TCR rearrangements31 suggest that early MRD negativity was related to lower risk of relapse and that they could achieve high survival rates without alloSCT. Persistence of MRD in children with Ph1 + ALL at later time points of therapy was associated with a higher incidence of disease relapse. [53] Similar results were observed in adult Ph1+ ALL patients. [32] The incidence of $\mathrm{Ph} 1+\mathrm{ALL}$ is $20-30 \%$ of adult patients with ALL. Achieving a deeper molecular response (RTqP$C R$ for $B C R-A B L 1$ transcripts with a limit of detection of 10-4 to 10-5) with intensive chemotherapy plus one TKI has been associated with superior outcomes, despite not undergoing alloSCT in first remission. [54] MRD has also been shown to predict outcomes in patients with Ph1+ ALL in a variety of situations, such as in patients undergoing regimens based on non-intensive induction therapy, including TKI plus corticosteroids, as well as TKI plus chemotherapy, whether or not followed by consolidation with alloSCT, according to age, molecular response, clinical eligibility and donor availability. [55,61] .In these series, the complete molecular remission achieved until 1 or 2 cycles of the induction therapy is associated to higher disease free survival (DFS) and lower CIR. $[55,61]$ On the other hand, based on MRD kinetics by the evaluation of BCR-ABL1 transcript, patients who underwent alloSCT in CR1, after chemotherapy plus dasatinib, showed a significant difference in DFS ( $p$ $=0.0018)$ and $\mathrm{CIR}(\mathrm{p}=0.0015)$ between early stable molecular responders (after 2 cycles of treatment) and poor molecular responders. However, there was no difference between early stable molecular responders and late molecular responders. [62]

The role of alloSCT is controversial with the resulting improvements seen by incorporating TKIs into first-line regimens for Ph1+ ALL. Although the therapy intensification with alloSCT still represents a good curative option, the introduction of novel approaches with ITK and immunotherapeutic agents is likely to improve the outcome of these patients further, and might mean that SCT can be avoided in a proportion of cases. [54,61] Nevertheless in any situation, MRD plays a role in guiding the best treatment choices.

Although several studies have shown the impact of molecular lesions on the ALL prognosis based on retrospective studies, it is difficult to incorporate this information into the MRD data to refine the prognosis in the face of therapeutic intensification. [30] Controlled studies can associate this information and establish treatment algorithms to improve the management of patients with ALL.

\section{T Immunophenotype ALL and MRD response}

T-ALL shows a slower blast clearance compared with BCP-ALL in the context of identical therapy, proving that they are biologically different diseases. The AIEOP-BFM 2000 protocol evaluated the impact of MRD by PCR in 464 T-ALL. This study showed that patients with MRD $<0.01 \%$ at the end of induction therapy has the most favorable prognosis, however, patients who became MRD negative by the end of consolidation also had a favorable outcome. [7] In contrast, patients who continued to show a high MRD level $(\geq 0.1 \%)$ at the end of consolidation phase had a high relapse risk. [7]

MRD is also prognostic in early T-cell precursor ETPALL, a more aggressive subset of T-ALL, which accounts for $15 \%$ of all T-cell ALL in children and 35\% in adult T-cell disease. It is also associated with high MRD levels post-induction therapy and also inferior long-term outcomes. $[63,64]$ The low frequency of this type of leukemia makes it difficult to guide treatment, although there is a consensus on more intensive treatment for this group of patients. [63] Therapy intensification, mainly based on high MRD status, resulted in a comparable outcome for ETP-ALL and non-ET-ALL patients. [65]

Adult T-ALL treatment groups demonstrated that patients who did not achieve molecular remission (MRD $>10-4$ ) after induction therapy have a lower survival rate than patients with MRD-negative $(<10-4)$. [64]

The GMALL group has reported a beneficial effect of alloSCT in patients with early and mature T immunophenotype, who had 10 years of OS of $25 \%$ without allo-SCT vs 59\% for those who underwent allo-SCT. [66]

Genetic lesions in T-ALL are diverse and complex, but their prognostic impact is not well defined and they are not widely used for risk stratification.39 Mutation of the NOTCH1/FBXW7 was found in at least $60 \%$ of adult patients with T-ALL, which has been described as a good-risk group with significantly higher OS and lower CIR rates in patients without PTEN or NK-RAS mutations. However, this result was not reproducible among the treatment groups and there are limitations in the use of these data for treatment decisions. [64]

\section{SAMPLES FOR MRD ASSESSMENT}

Bone marrow (BM) samples are preferable used for BCP-ALL instead of peripheral blood (PB) for ALL $M R D$, regardless of the method used, because the frequencies of BCP-ALL cells in paired $\mathrm{PB}$ and $\mathrm{BM}$ samples are significantly higher in $\mathrm{BM}$ than in $\mathrm{PB}$, ranging from 1 to 3 logs. On the other hand, a strong 
correlation can be observed between the frequencies of T-ALL cells in PB and BM, but the differences can occur up to 1 log, in favor of BM samples. [29]

\section{MRD ASSESSMENT REPORT}

To allow a correct interpretation of the MRD results, the MRD report must provide clear information about the MRD result and the MRD technique used, including the limits of detection and quantification achieved by the specific assay used, which are parameters of the sensitivity of the method. $[67,68]$

\section{CONCLUSION}

Relapse remains the main cause of treatment failure in patients with ALL who have undergone allogeneic SCT. Currently, MRD is the most important prognos-

\section{REFERENCES}

1. Cave H, van der Werff, ten Bosch J, Suciu S, Guidal C,Waterkeyn C, et al. Clinical significance of minimal residual disease in childhood acute lymphoblastic leukemia. European Organization for Research and Treatment of Cancer-Childhood Leukemia Cooperative Group. N Engl J Med.1998;339(9):591-8.2.

2.Zhou J, Goldwasser MA, Li A, Dahlberg SE, Neuberg $\mathrm{D}$, WangH, et al. Quantitative analysis of minimal residual disease predicts relapse in children with B-lineage acute lymphoblastic leukemia in DFCl ALL Consortium Protocol95-01. Blood. 2007;110(5):1607-11.3.

3. Borowitz MJ, Devidas M, Hunger SP, Bowman WP, Carroll AJ, Carroll WL, et al. Clinical significance of minimal residual disease in childhood acute lymphoblastic leukemia and its relationship to other prognostic factors: a Children'sOncology Group study. Blood. 2008;111(12):5477-85.

4. Basso G, Veltroni M, Valsecchi MG, Dworzak MN, Ratei $R$, Silvestri $D$, et al. Risk of relapse of childhood acute lymphoblastic leukemia is predicted by flow cytometric measurement of residual disease on day 15 bone marrow. J Clin Oncology 2009; 27(31): 5168-74.

5. Brüggemann $M$, Schrauder $A$, Raff $T$, Pfeifer $H$, tic parameter that can guide clinical decisions in this scenario. However, it is essential to have criteria to incorporate MRD results into clinical management Evaluation of each information discussed below and how the treatment used can impact the therapeutic response are crucial. Thus, a more accurate choice of a better treatment option for each ALL-patient can be made.

\section{CONFLICTS OF INTEREST}

Author declare no conflicts of interest

\section{ACKOWLEGEMENTS}

The author thanks Dr Vergilio Antonio Rensi Colturato for revising the text.

Dworzak M, Ottmann OG, et al. Standardized MRD quantification in European ALL trials: Proceedings of the Second International Symposium on MRD assessment in Kiel, Germany, 18-20September 2008. Leukemia. 2010;24(3):521-35.

6. Conter V, Bartram CR, Valsecchi MG, Schrauder A, Panzer-Grümayer R, Möricke A, et al. Molecular response to treatment redefines all prognostic factors in children and adolescents with B-cell precursor acute lymphoblastic leukemia: results in 3184 patients of the AIEOP-BFM ALL 2000 study. Blood 2010; 115(16): 3206-14.

7. Schrappe M, Valsecchi MG, Bartram CR, Schrauder A, Panzer-Grümayer R, Möricke $A$, et al. Late MRD response determines relapse risk overall and in subsets of childhood T-cell ALL: results of the AIEOP-BFM-ALL 2000 study. Blood. 2011; 118(8):2077-84.

8. Vora A, Goulden N, Wade R, Mitchell C, Hancock $J$, Hough $\mathrm{R}$, et al. Treatment reduction for children and young adults with low-risk acute lymphoblastic leukaemia defined by minimal residual disease (UKALL 2003): a randomised controlled trial. The Lancet Oncology 2013; 14(3): 199-209. 
9. Borowitz MJ, Wood BL, Devidas M, Loh ML, Raetz EA, Salzer WL, et al. Prognostic significance of minimal residual disease in high risk B-ALL: a report from Children's Oncology Group study AALL0232. Blood 2015; 126(8): 964-71

10. Pui $\mathrm{CH}$, Pei $\mathrm{D}$, Coustan-Smith $\mathrm{E}$, Jeha $\mathrm{S}$, Cheng C, Bowman WP et al. Clinical utility of sequential minimal residual disease measurements in the context of risk-based therapy in childhood acute lymphoblastic leukaemia: a prospective study. The Lancet Oncology 2015; 16(4): 465-74.

11. Pieters $\mathrm{R}$, de Groot-Kruseman $\mathrm{H}$, Van der Velden V, Fiocco $M$, van den Berg $H$, de Bront E et al. Successful Therapy Reduction and Intensification for Childhood Acute Lymphoblastic Leukemia Based on Minimal Residual Disease Monitoring: Study ALL10 From the Dutch Childhood Oncology Group. J Clin Oncology 2016; 34(22): 2591-601.

12. Gaipa G, Cazzaniga G, Valsecchi MG, Panzer-Grümayer R, Buldini B, Silvestri Det al. Time point-dependent concordance of flow cytometry and real-time quantitative polymerase chain reaction for minimal residual disease detection in childhood acute lymphoblastic leukemia.Haematologica. 2012;97(10):1582-1593.

13. Bar M, Wood BL, Radich JP, Doney KC, Woolfrey AE, Delaney C,Appelbaum FR, Gooley TA. Impact of Minimal Residual Disease, Detected by Flow Cytometry, on Outcome of Myeloablative Hematopoietic Cell Transplantation for Acute Lymphoblastic Leukemia Leukemia Research and Treatment 2014:1-9 http://dx.doi.org/10.1155/2014/42172

14. Eckert C, Hagedorn N, Sramkova L, Mann G, Panzer-Grümayer R, Peters $C$ et al. Monitoring minimal residual disease in children with high-risk relapses of acute lymphoblastic leukemia: prognostic relevance of early and late assessment. Leukemia (2015) 29, 1648-1655; doi:10.1038/leu.2015.59

15. Bader $\mathrm{P}$, Kreyenberg $\mathrm{H}$, Henze GHR, Eckert C, Reising $M$, Willasch A et al. Prognostic Value of Minimal Residual Disease Quantification Before Allogeneic Stem-Cell Transplantation in Relapsed Childhood Acute Lymphoblastic Leukemia: The ALL-REZ BFM Study Group. J Clin Oncol 2009; 27:377-384.

16. Shen Z, Gu X, Mao W, Yin L, Yang L, Zhang Z, et al. Influence of pre-transplant minimal residual disease on prognosis after Allo-SCT for patients with acute lymphoblastic leukemia: systematic review and meta-analysis BMC Cancer .2018; 18(755): 2-12 https://doi.org/10.1186/s12885-018-4670-5
17. Zhao XS, Liu YR, Zhu HH, et al. Monitoring MRD with flow cytometry: an effective method to predict relapse for ALL patients after allogeneic hematopoietic stem cell transplantation. Ann Hematol. 2012;91(2):183-192.

18. Bader $P$, Kreyenberg $H$, von Stackelberg A, Eckert C, Salzmann-Manrique E, Meisel R et al. Monitoring of Minimal Residual Disease After Allogeneic Stem-Cell Transplantation in Relapsed Childhood Acute Lymphoblastic Leukemia Allows for the Identification of Impending Relapse: Results of the ALL-BFM-SCT 2003 Trial. J Clin Oncol 2015 33:1275-1284

19. Bader P, Salzmann-Manrique E, Balduzzi A, Dalle $\mathrm{JH}$, Woolfrey $\mathrm{AE}$, Bar M, et al More precisely defining risk peri-HCT in pediatric ALL: pre- vs postMRD measures, serial positivity, and risk modeling. Blood advances 2019; 3(21): 3393-3405

20. Wood B, Wu D, Crossley B, Dai Y, Williamson D, Gaward $C$ et al. Measurable residual disease detection by high-throughput sequencing improves risk stratification for pediatric B-ALL. Blood 2018; 131(12): 1350-9.

21. Lovisa F, Zecca M, Rossi B, Campeggio M, Magrin $E$,Giarin $E$, et al. Pre- and post-transplant minimal residual disease predicts relapse occurrence in children with acute lymphoblastic leukaemia. British Journal of Haematology, 2018, 180, 680-693. doi: 10.1111/bjh.15086

22. Pulsipher MA, Carlson C, Langholz B, Wall DA, Schultz KR, Bunin $\mathrm{N}$ et al. IgH-V(D)J NGS-MRD measurement pre- and early post-allotransplant defines very low- and very high-risk ALL patients. Blood. 2015;125(22):3501-3508

23. Wang $X Y$, Fan $Q Z, X u L P$, Wang $Y$, Zhang $X H$, Chen $\mathrm{H}$ et al. The quantification od MRD pre and post-unmanipulated haploidentical allograft by multiparametric flow cytometry in pediatric Acute Lymphoblastic Leukemia. Cytometry B 2020; 98: 75-87

24. Terwey TH, Hemmati PG, Nagy M, Pfeifer H, Göbuget $N$, Brüggemann $M$ et al. Comparison of chimerism and minimal residual disease monitoring for relapse prediction after allogeneic stem cell transplantation for adult acute lymphoblastic leukemia. Biol Blood Marrow Transplant. 2014;20(10):1522-1529.

25. Nishiwaki S, Imai K, Mizuta S, Kanamori H, Ohashi $K$, Fukuda T, et al. Impact of MRD and TKI on allogeneic hematopoietic cell transplantation for $\mathrm{Ph}+\mathrm{ALL}$ : 
a study from the adult ALL WG of the JSHCT. Bone Marrow Transplant. 2016; 51(1):43-50.

26. Kotrova M, Muzikova K, Mejstrikova E, Novakova M, Bakardjjeva-Mihaylova $\mathrm{V}$, Fiser $\mathrm{K}$ et al. The predictive strength of next-generation sequencing MRD detection for relapse compared with current methods in childhood ALL. Blood. 2015;126(8):1045-1047.

27. Theunissen $P$, Mejstrikova $E$, Sedek $L$, van der Sluijs-Gelling AJ, Gaipa G, Bartels M, et al. Standardized flow cytometry for highly sensitive MRD measurements in B-cell acute lymphoblastic leucemia. Blood. 2017;129(3):347-357

28. Brüggemann $M$, Kotrova $M$. Minimal residual disease in adult ALL: technical aspects and implications for correct clinical interpretation. Blood advances. 2017;1(25):2456-2466 DOI 10.1182/ bloodadvances.2017009845.

29. van Dongen JJM, van der Velden VHJ, Brüggemann M, Orfao A Minimal residual disease diagnostics in acute lymphoblastic leukemia: need for sensitive, fast, and standardized technologies Blood. 2015;125(26):3996-4009

30. Short N, Jabbour E, Albitar M, Lima M, Gore L, Jorgensen J, Logan AC, Park J, Ravandi F, Shah B, Radich J, Kantarjian H. Recommendations for the Assessment and Management of Measurable Residual Disease in Adults With Acute Lymphoblastic Leukemia: A Consensus of North American Experts Am J Hematol. 2019; 94(2):257-265. doi: 10.1002/ajh.25338.

31. Jeha S, Coustan-Smith E, Pei D, Sandlund JT, Rubnitz JE, Howard SC, Inaba H, Bhojwani D, Metzger ML, Cheng C, Choi JK, Jacobsen J, Shurtleff SA, Raimondi S, Ribeiro RC, Pul CH, Campana D. Impact of tyrosine kinase inhibitors on minimal residual disease and outcome in childhood Philadelphia chromosome-positive acute lymphoblastic leukemia. Cancer.2014;120(10):1514-1519.

32. Lee S, Kim DW, Cho BS, Yoon JH, Shin SH, Yahng $\mathrm{SA}$, et al. Impact of minimal residual disease kinetics during imatinib-based treatment on transplantation outcome in Philadelphia chromosome-positive acute lymphoblastic leukemia. Leukemia 2012; 26(11): 2367-74.

33. Schrappe $M$. Minimal residual disease: optimal methods, timing, and clinical relevance for an individual patient. Hematology 2012: 1137-142
34. Garand R, Beldjord K, Cave H, Fossat C, Arnoux I, Asnafi V, et al. A Flow cytometry and IG/TCR quantitative $P C R$ for minimal residual disease quantitation in acute lymphoblastic leukemia: a French multicenter prospective study on behalf of the FRALLE, EORTC and GRAALL. Leukemia 2013; 27: 370-376

35. Theunissen PMJ, Sedek L, De Haas V, Szczepanski T,Van Der Sluijs A, Mejstrikova E, et al. Detailed immunophenotyping of B-cell precursors in regenerating bone marrow of acute lymphoblastic leukaemia patients: implications for minimal residual disease detection. British Journal of Haematology, 2017; 178: 257-266. doi: 10.1111/bjh.14682

36. Cherian S, Miller V, McCullouch V, Dougherty K, Fromm JR, Wood BL. A Novel Flow Cytometric Assay for Detection of Residual Disease in Patients with B-Lymphoblastic Leukemia/Lymphoma Post Anti-CD19 Therapy. Cytometry B 2018;94(1):112120 doi: $10.1002 /$ cyto.b. 21482 .

37. Pui $\mathrm{CH}$, Pei D, Raimondi SC, Coustan-Smith $\mathrm{E}$, Jeha S, Cheng C, et al. Clinical Impact of Minimal Residual Disease in Children with Different Subtypes of Acute Lymphoblastic Leukemia Treated with Response-Adapted Therapy. Leukemia. 2017; 31(2): 333-339. doi:10.1038/leu.2016.234.

38. O'Connor D, Enshaei A, Bartram J, Hancock J, Harrison CJ, Hough R, et al. Genotype-Specific Minimal Residual Disease Interpretation Improves Stratification in Pediatric Acute Lymphoblastic Leukemia. J Clin Oncol 2017; 36:34-43.

39. Bhojwani D, Yang JJ, Pui CH Biology of Childhood Acute Lymphoblastic Leukemia Pediatr Clin North Am. 2015 ;62(1): 47-60. doi:10.1016/j. pcl.2014.09.004.

40. Beldjord K, Chevret S, Asnafi V, Huguet F, Boulland ML, Leguay $T$ et al. Oncogenetics and minimal residual disease are independent outcome predictors in adult patients with acute lymphoblastic leukemia. Blood 2014; 123(24): 3739-49.

41. Gupta SK, Bakhshi S, Chopra A, Kamal VK. Molecular genetic profile in BCR-ABL1 negative pediatric B-cell acute lymphoblastic leukemia can further refine outcome prediction in addition to that by end-induction minimal residual disease detection. Leukemia \& lymphoma 2017: 1-6.

42. McNeer JL, Devidas M, Dai Y, Carroll AJ, Heerema NA, Gastier-Foster JM et al. Hematopoietic StemCell Transplantation Does Not Improve the Poor 
Outcome of Children With Hypodiploid Acute Lymphoblastic Leukemia: A Report From Children's Oncology Group. J Clin Oncol 2019 37:780789.

43. Meyer C, Burmeister T, Gröger D, Tsaur G, Fechina $L$, Renneville $A$, et al. The MLL recombinome of acute leukemias in 2017. Leukemia 2018; 32: 273-284

44. Roberts KG, Li Y, Payne Turner D, Harvey RC, Yang YL, Pei $D$, et al. Targetable kinase activating lesions in Ph-like acute lymphoblastic leukemia of BCR-ABL1-like acute lymphoblastic leukemia. N Engl J Med 2014; 371:1005-15. DOI: 10.1056/NEJMoa1403088

45. Weston BW, Hayden MA, Roberts KG, Bowyer S, Hsu J, Fedoriw $G$ et al. Tyrosine kinase inhibitor therapy induces remission in a patient with refractory EBF1-PDGFRB-positive acute lymphoblastic leukemia. J Clin Oncol. 2013; 31:e413-e416.

46. Roberts KG,Yang WL, Payne-Turner D, Lin W, Files $\mathrm{JK}$, Dickerson $\mathrm{K}$, et al. Oncogenic role and therapeutic targeting of ABL-class and JAK-STAT activating kinase alterations in Ph-like ALL Blood advances 2017; 1(20) 1657-1671 2017. DOI 10.1182/ bloodadvances.2017011296.

47. Hunger SP, Mullighan CG. Redefining ALL classification: toward detecting high-risk ALL and implementing precision medicine. Blood. 2015 Jun 25; 125(26): 3977-3987. doi: 10.1182/ blood-2015-02-580043

48. Roberts KG, Pei D, Campana D, Payne-Turner D, Li Y, Cheng C, et al; Outcomes of Children With BCRABL1-Like Acute Lymphoblastic Leukemia Treated With Risk-Directed Therapy Based on the Levels of Minimal Residual Disease. J Clin Oncol. 2014; 32:3012-3020. DOI: 10.1200/JCO.2014.55.4105

49. Stanulla $M$, Dagdan E, Zaliova $M, M$ *oricke $A$, Palmi C, Cazzaniga $G$ et al.. IKZF1plus Defines a New Minimal Residual Disease-Dependent VeryPoor Prognostic Profile in Pediatric B-Cell Precursor Acute Lymphoblastic Leukemia. J Clin Oncol 2018; 36: 1-10. DOI: https://doi.org/10.1200/ JCO.2017.74.3617

50. Moorman AV, Robinson $H$, Schwab C, Richards SM, Hancock J, D Mitchell C, et al: Risk-directed treatment intensification significantly reduces the risk of relapse among children and adolescents with acute lymphoblastic leukemia and intrachromosomal amplification of chromosome 21: A comparison of the MRC ALL97/99 and UKALL2003 trials. J Clin Oncol 2013; 31:3389-3396.

51. Heerema NA, Carroll AJ, Devidas M, Loh ML, Borowitz MJ, Gastier-Foster JM, et al. Intrachromosomal amplification of chromosome 21 is associated with inferior outcomes in children with acute lymphoblastic leukemia treated in contemporary standard-risk Children's Oncology Group studies: A report from the Children's Oncology Group. J Clin Oncol 2013;31:3397-3402

52. Attarbaschi A, Panzer-Grumayer R, Mann G, Möricke A, König M, Mecklenbräueker A, et al: Minimal residual disease-based treatment is adequate for relapse-prone childhood acute lymphoblastic leukemia with an intrachromosomal amplification of chromosome 21: The experience of the ALLBFM 2000 trial. Klin Padiatr . 2014; 226:338-343

53. Cazzaniga G, Lorenzo P, Alten J, Röttgers S, Hancock J, Saha V, et al.. Predictive value of minimal residual disease in Philadelphia-chromosome-positive acute lymphoblastic leukemia treated with imatinib in the European intergroup study of post-induction treatment of Philadelphia-chromosome-positive acute lymphoblastic leukemia, based on immunoglobulin/T-cell receptor and $B C R / A B L 1$ methodologies. Haematologica 2018;103(1):107-115

54. Short NJ, Jabbour E, Sasaki K, Patel K, O'Brien SM, Cortes JE, et al. Impact of complete molecular response on survival in patients with Philadelphia chromosome-positive acute lymphoblastic leukemia. Blood 2016; 128(4): 504-7.

55. Ribera JM, Oriol A, González M, Vidriales B, Brunet $S$, Esteve J, et al. Concurrent intensive chemotherapy and imatinib before and after stem cell transplantation in newly diagnosed Philadelphia chromosome-positive acute lymphoblastic leukemia. Final results of the CSTIBES02 trial. Haematologica. 2010;95(1):87-95.DOI: 10.3324/haematol.2009.011221

56. Foà R, Vitale A, Vignetti M, Meloni G, Guarini A, Propris MS, et al. Dasatinib as first-line treatment for adult patients with Philadelphia chromosome positive acute lymphoblastic leukemia. Blood. 2011;118(25):6521-6528. https://doi.org/10.1182/ blood-2011-05-351403

57. Jabbour E, Kantarjian H, Ravandi F, Huang X, Daver $N$, DiNardo CD, et al. Combination of hyper-CVAD with ponatinib as first-line therapy for patients with Philadelphia chromosome-positive acute lympho- 
blastic leukaemia: a single-centre, phase 2 study. Lancet Oncol. 2015;16(15):1547-1555.

58. Chalandon Y, Thomas X, Hayette S, Cayuela JM, Abbal $C$, Huguet $F$, et al. Group for Research on Adult Acute Lymphoblastic Leukemia (GRAALL). Randomized study of reduced-intensity chemotherapy combined with imatinib in adults with Ph-positive acute lymphoblastic leukemia. Blood.2015;125(24):3711-3

59. Rousselot P, Coudé MM, Gokbuget N, Passerini CG, Hayette S, Cayuela JM, et al. Dasatinib and low-intensity chemotherapy in elderly patients with Philadelphia chromosome-positive ALL. Blood. 2016;128(6):774-782. doi: 10.1182/ blood-2016-02-700153

60. Chiaretti S, Vitale A, Vignetti M, Piciocchi A, Fazi, $P$, Elia $L$, et al. A sequential approach with imatinib, chemotherapy and transplant for adult $\mathrm{Ph}+$ acute lymphoblastic leukemia: final results of the GIMEMA LAL 0904 study. Haematologica 2016;101(12):1544-1552

61. Short NJ, Jabbour E, Chiaretti S. Should Treatment of Philadelphia Chromosome-Positive Acute Lymphoblastic Leukemia Be Intensive? Clinical Advances in Hematology \& Oncology 2016;14 (11): 893-896

62. Yoon JH, Yhim HY, Kwak JY, Ahn JS, Yang DH, Lee $\mathrm{JJ}$, et al. Minimal residual disease-based effect and long-term outcome of first-line dasatinib combined with chemotherapy for adult Philadelphia chromosome-positive acute lymphoblastic leukemia. Annals of Oncology 27: 1081-1088, 2016 doi:10.1093/annonc/mdw123
63. Jain N, Lamb AV, O'Brien S, Ravandi F, Konopleva $M$, Jabbour E, et al . Early T-cell Precursor Acute Lymphoblastic Leukemia/Lymphoma (ETP-ALL/ LBL) in Adolescents and Adults: A High-Risk Subtype. Blood. 2016; 14;127(15):1863-9. doi: 10.1182/ blood-2015-08-661702.

64 .Marks DI, Rowntree C. Management of adults with T-cell lymphoblastic leukemia .Blood. 2017;129(9):1134-1142)

65. Athale UH, Gibson PJ, Bradley NM, Malkin DM, MD, Hitzler J, Minimal Residual Disease and Childhood Leukemia: Standard of Care Recommendations From the Pediatric Oncology Group of Ontario MRD Working Group. Pediatr Blood Cancer 2016;63:973-982

66. Hoelzer D, Thiel E, Arnold R, Beck J, Beelen DW, Bornhäuser $M$, et al. Successful Subtype Oriented Treatment Strategies in Adult T-All; Results of 744 Patients Treated in Three Consecutive GMALL Studies. Blood (2009) 114 (22): 324. https://doi. org/10.1182/blood.V114.22.324.324

67. Arroz M, Came N, Lin P, Chen W, Yuan C, Lagoo A, et al. Consensus Guidelines on Plasma Cell Myeloma Minimal Residual Disease Analysis and Reporting. Cytometry Part B 2016; 90B: 31-39.

68. Clinical and Laboratory Standards Institute (CLSI). Evaluation of Detection Capability for Clinical Laboratory Measurement Procedures; Approved Guideline, 2nd ed. CLSI document EP17-A2 (ISBN 1-56238-795-2). Pennsylvania: Clinical and Laboratory Standards Institute; 2012. 